\title{
Obstructive sleep apnoea and metabolic syndrome: put CPAP efficacy in a more realistic perspective
}

\author{
Jean-Louis Pépin, ${ }^{1,2}$ Renaud Tamisier, ${ }^{1,2}$ Patrick Lévy ${ }^{1,2}$
}

Non-communicable Diseases, mainly cardiovascular diseases, cancer, diabetes and chronic respiratory diseases, are responsible for two-thirds of the 57 million annual deaths worldwide. ${ }^{1}$ Obesity and obstructive sleep apnoea (OSA) are among the key players involved in noncommunicable diseases. Several studies have reported an independent association of OSA with the different components of the metabolic syndrome, particularly hypertension, insulin resistance and abnormal lipid metabolism. ${ }^{2}$ Both OSA and metabolic syndrome are associated with an increase risk in cardiovascular disease. Hence, rapidly accumulating data from both epidemiological and clinical studies $^{3}$ have suggested that OSA is independently associated with alterations in glucose metabolism and with an increased risk of developing type 2 diabetes. Indeed, recent reports have indicated that more than $50 \%$ of patients with type 2 diabetes have OSA. Multiple mechanistic pathways contribute to the deteriorated plasma glucose/insulin homeostasis in OSA, the number one being sympathetic over activity, ${ }^{4}$ due to sleep fragmentation and intermittent hypoxia (figure 1). Independently of autonomic nervous system activation, in animal models intermittent hypoxia contributes to decreased glucose utilisation in oxidative muscle fibres. ${ }^{5}$ Intermittent hypoxia also seems to be responsible for increased beta-cell proliferation and cell death, the latter being due to oxidative stress. ${ }^{6}$ In fat tissue, chronic intermittent hypoxia $(\mathrm{CIH})$ the hallmark of OSA exacerbate adipose tissue inflammation and lead to a dysregulated production of

'Laboratoire HP2, Hypoxie: Physiopathologies, Faculté de Médecine, Université Joseph Fourier; INSERM U 1042, Grenoble Cedex 09, France

2Pôle Locomotion, Rééducation et Physiologie, CHU de Grenoble, Grenoble Cedex 09, France

Correspondence to Professor Jean Louis Pépin, Laboratoire EFCR, CHU de Grenoble, BP217X, Grenoble Cedex 09 38043, France; jpepin@chu-grenoble.fr adipocytokines which may contribute to insulin resistance and could trigger nonalcoholic fatty liver disease (figure 1). We have demonstrated in morbid obesity that $\mathrm{CIH}$ is strongly associated with higher systemic inflammation (IL-6) and more severe fibrotic or inflammatory liver injuries. ${ }^{7}$ Visceral fat releases free fatty acids in the portal vein leading to accumulation of hepatic fat and development of hepatic insulin resistance. Regarding lipid abnormalities, intermittent hypoxia leads to an increase in serum cholesterol, to an up-regulation of triglycerides and phospholipids biosynthesis, and to an inhibition of triglycerides uptake in the liver. $\mathrm{CIH}$ is also associated with lipoprotein lipase inhibition in the adipose tissue which leads to an increase in plasma chylomicrons and very low-density lipoprotein cholesterol (VLDL-c) in the bloodstream that may favour the progression of atherosclerosis. ${ }^{8}$

In summary, $\mathrm{CIH}$, the main hallmark of OSA is assumed to have a deleterious impact on glucose and lipid homeostasis. However, it is crucial to understand that the impact of $\mathrm{CIH}$ will differ depending on the weight status both in humans and in animals exposed to intermittent hypoxia. Obesity per se is associated with striking increase in inflammatory markers possibly overwhelming the additional effects of intermittent hypoxia exposure. For instance, $\mathrm{CIH}$ induced non-alcoholic fatty liver disease in diet-induced obesity mice which was not observed in lean mice. 9 Accordingly, obesity-related inflammation is not significantly augmented by $\mathrm{CIH}^{10}$ Such observations in animal studies may be expended to humans providing a potential explanation for the relative impact of continuous positive airway pressure (CPAP) treatment on the cardiometabolic risk profile in the presence of obesity.

In the recent study of Sharma et al, ${ }^{11}$ 86 OSA patients were randomly assigned to undergo 3 months of therapeutic CPAP followed by 3 months of sham
CPAP, in a cross over design, with a 1-month washout period. CPAP therapy lowered blood pressure and partially reversed metabolic abnormalities. There was a significant reduction in glycated haemoglobin, triglycerides, total cholesterol after effective CPAP as compared with sham CPAP, as well as a significant increase in the high-density lipoprotein (HDL) to total cholesterol ratio. The frequency of the metabolic syndrome was reduced after CPAP (reversal found in $13 \%$ of patients undergoing CPAP versus $1 \%$ in those undergoing sham CPAP). Concomitantly, there were significant decreases in body mass index (BMI) and visceral and subcutaneous fat in the CPAP arm. Although 547 patients were assessed for eligibility in this study, 360 were excluded as receiving drugs for hypertension, diabetes mellitus, or dyslipidaemia. Also, 256 had pre-existing cardiovascular disease, nephropathy, or diabetic retinopathy and were discarded. At the end, the 90 randomised patients represented a highly selected obstructive sleep apnoea population. Hoyos and colleagues $^{12}$ strongly challenge these data and the reversibility of metabolic anomalies observed in OSA patients treated with CPAP. The authors conducted a randomised sham-controlled study in 65 nondiabetic CPAP-naive men with moderate to severe OSA. Patients were randomised to receive either real $(n=34)$ or sham $(\mathrm{n}=31)$ CPAP for 12 weeks. At 12-weeks, all subjects received real CPAP for an additional 12 weeks. Main outcomes were the change from baseline to week 12 in insulin sensitivity (ISx), visceral abdominal fat (VAF) and liver fat. There were no between-group differences at 12 weeks in ISx, VAF, liver fat or any other cardio-metabolic parameter. At 24 weeks, ISx, but not VAF or liver fat, were improved compared with baseline. Although the study was conducted in OSA patients patient free of type 2 diabetes, patients having other cardiometabolic co-morbidities and being treated by antihypertensive and lipid lowering drugs were not systematically excluded from randomisation. The study of Hoyos et al. was designed as a practical, intention to treat, study that could be more applicable to a typical OSA population. The negative results are in accordance with two previous randomised controlled trials evaluating metabolic outcomes with therapeutic or sham CPAP in patients with $^{13}$ and without diabetes. ${ }^{14}$ In both studies assessing obese OSA patients, CPAP did not change glucose, lipids, insulin resistance level or the ratio of 


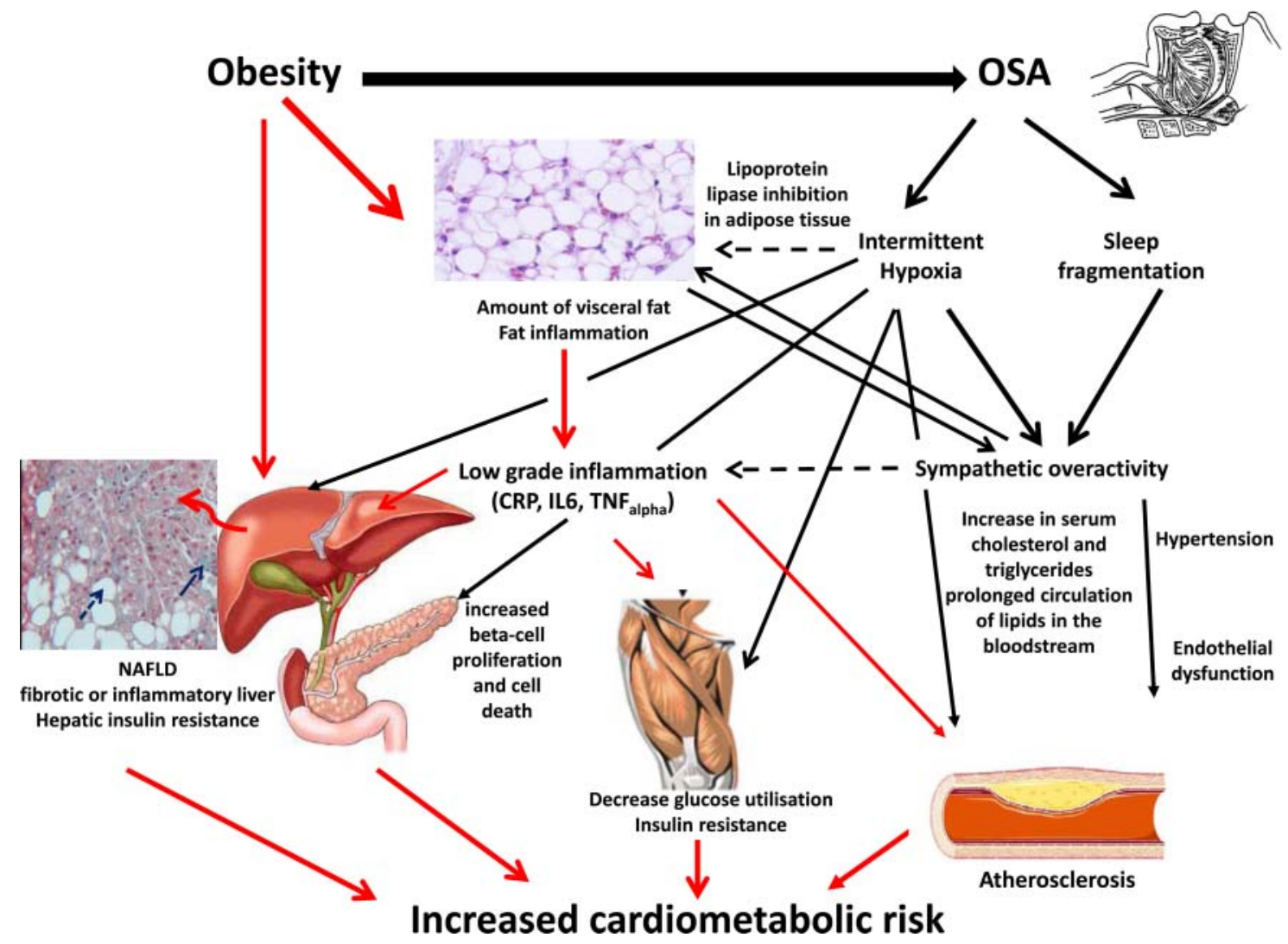

Figure 1 Obesity and sleep apnoea are sharing common mechanistic pathways to increase cardiometabolic risk. CRP, C reactive protein; IL-6, interleukin 6; NAFLD, non-alcoholic fatty liver disease; OSA, obstructive sleep apnoea; TNF- $\alpha$, tumour necrosis factor $\alpha$.

patients with metabolic syndrome. In obese sleep apnoea individuals insulin sensitivity is likely to be determined primarily by obesity and, to a lesser extent, by sleep apnoea. ${ }^{15}$ Accordingly, a recent meta-analysis did not show a significant impact of CPAP treatment on glucose homeostasis. ${ }^{16}$

Uncontrolled studies have shown a beneficial effect of CPAP on lipid abnormalities but there are more discrepancies in controlled studies with some being negative $^{14}$ whilst others found improvement. Part of the recovery in lipid metabolism could be explained by the concomitant weight loss ${ }^{11}$ as suggested by the correlation between the metabolic syndrome and the change in BMI during the Sharma's study. Postprandial lipid level is now recognised as a strong marker of cardiovascular risk. Recently, Phillips and colleagues used seven blood samples drawn across both the wake and sleep periods to demonstrate in a randomised trial that CPAP improved postprandial triglycerides and mean 24-h total cholesterol levels. ${ }^{17}$

Part of the differences in the metabolic dysfunction response to CPAP could be explained by changes in visceral fat. The Hoyos's negative study is important in suggesting that reduction in VAF and liver fat, as central components of the metabolic syndrome, cannot be achieved with CPAP alone. Another study from the same group ${ }^{18}$ using MRI technique for assessing visceral and liver fat also failed to demonstrate any improvement. In the Sharma study, to explain the significant improvement in visceral fat, the authors argued that this could be related to a consequent increase in physical activity after CPAP. This is unlikely to be the case as, in the only available RCT, West et al ${ }^{19}$ have demonstrated that obese patients with OSA do not actually increase their physical activity after CPAP treatment.

Another explanation for the heterogeneity in CPAP response is related to the severity of the disease. In the Sharma study, subgroups with more severe metabolic derangements at baseline had greater treatment responses. ${ }^{11}$ As the patients in their study were naïve of any treatment of cardiometabolic risk and had more severe metabolic anomalies at baseline, this could be one reason for the better response to CPAP therapy. Another recent RCT did not show that impaired glucose tolerance normalised after CPAP. However, insulin sensitivity improved in those with $\mathrm{AHI} \geq 30$, suggesting beneficial metabolic effects of CPAP only in severe sleep apnoea. ${ }^{20}$ The MOSAIC randomised controlled trial $(n=391)^{21}$ conducted in minimally symptomatic mild to moderate OSA (median oxygen desaturation index: $10 / h$ ) showed that CPAP compared with standard care improves sleepiness but not calculated vascular risk. The treatment effect of CPAP on lipids and glucose control indices was not significant. The only significant effect was a small fall in obesity indices (BMI, waist circumference) favouring standard care.

A major issue is also the optimal duration for CPAP use. There is little data to establish a clear threshold of CPAP use reducing cardio-metabolic risks. It has been suggested from meta-analysis that there is a dose-response relationship between the obtained reduction in blood pressure and nightly usage of the device. $^{22}$ Hoyos et al showed in figure 6 in their article that there was no relationship between CPAP adherence and change in VAF and no effect was found even in high CPAP compliers.

In summary, there are data supporting an independent role of OSA in metabolic dysfunction. ${ }^{23}$ Effective treatment of OSA has thus been proposed as an important 
target for improving cardio-metabolic risk. The Hoyos paper suggests that it is not realistic to expect a clinically relevant decrease in VAF with CPAP therapy. In unselected OSA, there is only a fringe benefit of CPAP on metabolic dysfunction. Potentially, only the severe patients, without morbid obesity and under the condition of high CPAP usage would respond. Moreover, the range of response is not equivalent to lipid lowering drugs or weight loss programmes. Whereas the majority of respiratory physicians limit their intervention to prescribing CPAP, this study emphasises the need to offer multiple modalities of treatment to OSA patients if their cardiovascular and metabolic risk profile is to be successfully modified. Rehabilitation programmes as well as weight loss reduction programmes should probably be implemented. Interestingly, men with sleep apnoea syndrome at baseline appear to exhibit smaller reduction in body weight and less metabolic improvements associated with the lifestyle intervention programme than men without sleep apnoea syndrome. ${ }^{24}$ Further studies may thus address whether CPAP treatment should be administered during the period of the lifestyle intervention programme in order to increase body weight loss and to improve the cardiometabolic risk markers.

Red arrows are corresponding to pathways mainly related to obesity that can be modified by lifestyle interventions but are not really affected by OSA treatment. It is then expected that CPAP alone could not achieve significant reduction of cardiovascular risk in obese OSA patients. There are needs for combined therapeutic strategies.

\section{Competing interests None.}

Provenance and peer review Not commissioned; internally peer reviewed.

Thorax 2012;67:1025-1027.

doi:10.1136/thoraxjnl-2012-202807

\section{REFERENCES}

1. The L. Time for action in New York on non-communicable diseases. Lancet 2011;378:961.

2. Levy P, Bonsignore MR, Eckel J. Sleep, sleep-disordered breathing and metabolic consequences. Eur Respir J 2009;34:243-60.

3. Morselli LL, Guyon A, Spiegel K. Sleep and metabolic function. Pflugers Arch 2012;463:139-60.

4. Tamisier R, Pepin JL, Remy J, et al. 14 nights of intermittent hypoxia elevate daytime blood pressure and sympathetic activity in healthy humans. Eur Respir J 2011;37:119-28.

5. liyori $\mathbf{N}$, Alonso LC, Li J, et al. Intermittent hypoxia causes insulin resistance in lean mice independent of autonomic activity. Am J Respir Crit Care Med 2007;175:851-7.

6. Xu J, Long Y-S, Gozal D, et al. $\beta$-cell death and proliferation after intermittent hypoxia: Role of oxidative stress. Free Radic Biol Med 2009;46:783-90.

7. Aron-Wisnewsky J, Minville C, Tordjman J, et al. Chronic intermittent hypoxia is a major trigger for non-alcoholic fatty liver disease in morbid obese. J Hepatol 2012;56:225-33

8. Drager L, Polotsky V. Lipid Metabolism: A New Frontier in Sleep Apnea Research. Am J Respir Crit Care Med 2011;184:288-90.

9. Drager LF, Li J, Reinke C, et al. Intermittent Hypoxia Exacerbates Metabolic Effects of Diet-Induced Obesity. Obesity 2011;19:2167-74.

10. Reinke C, Bevans-Fonti S, Drager LF, et al. Effects of different acute hypoxic regimens on tissue oxygen profiles and metabolic outcomes. J Appl Physiol 2011;111:881-90.

11. Sharma SK, Agrawal S, Damodaran D, et al. CPAP for the metabolic syndrome in patients with obstructive sleep apnea. N Engl J Med 2011;365:2277-86.

12. Hoyos CM, Killick R, Yee BJ, et al. Cardiometabolic changes after continuous positive airway pressure for obstructive sleep apnoea: a randomised shamcontrolled study. Thorax. Published Online First: 5 May 2012. doi:10.1136/thoraxjnl-2011-201420.

13. West SD, Nicoll DJ, Wallace TM, et al. Effect of $\mathrm{CPAP}$ on insulin resistance and $\mathrm{HbA} 1 \mathrm{c}$ in men with obstructive sleep apnoea and type 2 diabetes. Thorax 2007;62:969-74.
14. Coughlin SR, Mawdsley L, Mugarza JA, et al. Cardiovascular and metabolic effects of CPAP in obese males with OSA. Eur Respir J 2007;29:720-7.

15. Harsch IA, Schahin SP, Radespiel-Troger M, et al. Continuous positive airway pressure treatment rapidly improves insulin sensitivity in patients with obstructive sleep apnea syndrome. Am J Respir Crit Care Med 2004;169:156-62.

16. Hecht L, Mohler R, Meyer G. Effects of CPAP-respiration on markers of glucose metabolism in patients with obstructive sleep apnoea syndrome: a systematic review and meta-analysis. Ger Med Sci 2011;9:Doc20.

17. Phillips CL, Yee BJ, Marshall NS, et al. Continuous positive airway pressure reduces postprandial lipidemia in obstructive sleep apnea. Am J Respir Crit Care Med 2011;184:355-61.

18. Sivam S, Phillips CL, Trenell Ml, et al. Effects of 8 weeks of continuous positive airway pressure on abdominal adiposity in obstructive sleep apnoea. Eur Respir J 2012;40:913-18.

19. West SD, Kohler M, Nicoll DJ, et al. The effect of continuous positive airway pressure treatment on physical activity in patients with obstructive sleep apnoea: A randomised controlled trial. Sleep Med 2009;10:1056-8.

20. Weinstock TG, Wang X, Rueschman M, et al. A controlled trial of CPAP therapy on metabolic control in individuals with impaired glucose tolerance and sleep apnea. Sleep 2012;35:617-25B.

21. Craig S, Kohler M, Nicoll DJ, et al. Continuous Positive Airway Pressure (CPAP) improves sleepiness but not calculated vascular risk in patients with minimally symptomatic obstructive sleep apnoea (OSA); the MOSAIC randomised controlled trial. Thorax Published Online First: 30 Oct 2012. doi:10.1136/thoraxjnl-2012-202178

22. Haentjens $\mathbf{P}$, Van Meerhaeghe A, Moscariello A, et al. The impact of continuous positive airway pressure on blood pressure in patients with obstructive sleep apnea syndrome: evidence from a meta-analysis of placebo-controlled randomized trials. Arch Intern Med 2007;167:757-64.

23. Lam JC, Mak JC, Ip MS. Obesity, obstructive sleep apnoea and metabolic syndrome. Respirology 2012;17:223-36.

24. Borel AL, Leblanc $X$, Almeras N, et al. Sleep apnoea attenuates the effects of a lifestyle intervention programme in men with visceral obesity. Thorax 2012;67:735-41. 